\title{
The Local Time Distribution of a Particle Diffusing on a Graph
}

\author{
Alain Comtet ${ }^{1,2}$, Jean Desbois ${ }^{1}$ and Satya N. Majumdar ${ }^{3}$
}

2nd November 2018

\author{
${ }^{1}$ Laboratoire de Physique Théorique et Modèles Statistiques, Université Paris-Sud, Bât. 100, F-91405 \\ Orsay Cedex, France. \\ ${ }^{2}$ Institut Henri Poincaré, 11 rue Pierre et Marie Curie, 75005 Paris, France. \\ ${ }^{3}$ Laboratoire de Physique Quantique, Université Paul Sabatier, 31062 Toulouse Cédex, France.
}

\begin{abstract}
We study the local time distribution of a Brownian particle diffusing along the links on a graph. In particular, we derive an analytic expression of its Laplace transform in terms of the Green's function on the graph. We show that the asymptotic behavior of this distribution has non-Gaussian tails characterized by a nontrivial large deviation function.
\end{abstract}

Graphs are ubiquitous and fascinating objects [1]. In equilibrium statistical physics, the study of various model systems on graphs have provided deeper insights into how thermodynamic properties depend on the geometry of the underlying graph or network. For example, the study of a spin system on a complete graph provides an understanding of the thermodynamical properties at a mean field level. These studies on graphs are often nontrivial, the famous example being the Sherrington-Kirkpatrick model of Ising spin glasses defined on a complete graph [2]. More recently, models of nonequilibrium statistical physics such as the abelian sandpile model have also been studied on graphs providing deep insights to the dynamics [3]. The simplest dynamical example that has evoked enormous interest in both the physics and the mathematics community is the study of random walks on graphs, where a particle hops from one vertex to another provided they are connected by an edge. This has led to the study of the spectral properties of the discrete Laplacian operator on both regular [4] and random graphs. All these systems mentioned above share one common property: the links between vertices on the graph do not play an active role in the actual physical process, their only purpose is to provide just a connection between two vertices. For example, the Euclidean length of a link is completely irrelevant and the physical properties only depend on parameters such as the coordination number of vertices etc. Due to this passive nature of the links, we refer to such networks as 'passive-link' networks.

On the other hand, there exist 'active-link' networks where the links participate directly in the actual physical process. Examples include the networks for supplying household utilities such as electricity, water, telephone etc, the network of pipelines carrying oil and natural gases, the network of blood vessels in a living organism and many others. Quantum transport through mesoscopic networks provides yet another example of such 'active-link' network which has been studied experimentally and theoretically [5, 10]. Unlike their passive counterparts, the properties of physical observables on 'active-link' networks do depend on the Euclidean lengths of the links 
in addition to other parameters of the graph. Given the fact that the study of random walks on 'passive-link' networks has evoked so much interest and found numerous applications, it is natural to extend these studies on 'active-link' networks. In contrast to the 'passive-link' networks, the random walker does not hop between vertices of an 'active-link' network but actually undergoes continuous time Brownian motion along the links. A biological example of such a diffusion process on an 'active-link' network is the spread of an infectious virus or bacteria along the blood vessels in a living organism.

In this Letter we study analytically the properties of a particular physical observable associated with the diffusion process on an 'active-link' graph, namely the probability distribution of the local time spent by the Brownian particle at a given point on the graph. This quantity characterizes the amount of time $T$ spent by the particle between 0 and $t$ in the vicinity of a point. The corresponding quantity for a discrete random walk on a 'passive-link' network is the widely studied 'number of returns' to a given vertex. In the biological context mentioned above, the local time $T$ denotes the time spent by the diffusing virus in the vicinity of a point (e.g, near the brain or the lungs) within its own lifetime $t$ and hence is a measure of the damage that the virus can cause at a particular place in the network of blood vessels. Thus the study of the statistical properties of the local time in this context is important for medical purposes.

For a fixed total time $t$, the local time $T$ at any given point on the graph is clearly a random variable taking a different value for each history of the diffusion process. In this Letter we study analytically the probability distribution $P(T, t)$ of the local time and show that for any generic graph where the total lengths of the links is finite, the distribution has the generic asymptotic behavior, $P(T, t) \sim \exp [-t \Phi(T / t)]$ in the scaling limit $T \rightarrow \infty, t \rightarrow \infty$ but keeping the ratio $T / t$ fixed. The function $\Phi(x)$ is a large deviation function that characterize the non-Gaussian tails of this distribution. We provide a general formula for this large deviation function $\Phi(x)$ and calculate it explicitly in few specific examples.

Let us begin by establishing our notation.

Consider a graph $\mathcal{G}$ consisting of a set of $V$ vertices, labelled from 0 to $V-1$ and linked by $B$ links of finite lengths. The coordination of vertex $\alpha$ is denoted by $m_{\alpha}$, therefore $\sum_{\alpha=0}^{V-1} m_{\alpha}=2 B$.

Each link $[\alpha \beta]$ of length $l_{\alpha \beta}$ is identified with an interval $\left[0, l_{\alpha \beta}\right]$ of $R$. We denote by $x_{\alpha \beta}$ the coordinate on the link $[\alpha \beta]$ starting from vertex $\alpha$. Unless otherwise stated the total length of the graph $l=\sum_{[\alpha \beta]} l_{\alpha \beta}$ is assumed to be finite.

Now, let us consider a Brownian particle starting at $t=0$ from some point $O$. Without loss of generality we may assume that this point is a vertex $(\alpha=0)$ of the graph. As time evolves the particle will diffuse along the links and explore the whole graph. If the diffusion is recurrent (which is, in particular, the case for a finite graph), it will revisit infinitely often the initial vertex. The aim of this letter is to compute the probability distribution of the total time $T$ spent at $O$ between 0 and $t$. If $x(t)$ denotes the position of the particle at time $t$, the local time $T$ spent by this particle in an infinitesimal neighbourhood of $O$ may be defined as

$$
T \equiv \int_{0}^{t} \delta\left(x\left(t^{\prime}\right)\right) \mathrm{d} t^{\prime}
$$

where $x=0$ is the location of $\mathrm{O}$.

Let $P(T, t)$ be the probability distribution of $T$. This distribution was studied recently in the one dimensional Sinai type models and it was shown that the characteristic function of the local time distribution can be expressed in terms of the evolution of a quantum Hamiltonian by using a path integral or equivalently a backward Fokker-Planck approach [6]. This method can be easily extended to the present case where one dimensional lines connect up to form the 
'active-link' network. We find

$$
E\left(e^{-p T}\right) \equiv \int_{0}^{\infty} \mathrm{d} T P(T, t) e^{-p T}=\int_{\text {Graph }} \mathrm{d} x\left\langle x\left|e^{-t H}\right| 0\right\rangle,
$$

where $E$ denotes the expectation with respect to all Brownian paths over time $t$ each starting from $O$ and $H$ is a quantum Hamiltonian defined below. A further Laplace transform in $t$ gives

$$
\mathcal{L}=\int_{0}^{\infty} \mathrm{d} t e^{-\gamma t} E\left(e^{-p T}\right)=\int_{\text {Graph }} \mathrm{d} x\left\langle x\left|\frac{1}{H+\gamma}\right| 0\right\rangle \equiv \int_{\text {Graph }} \mathrm{d} x G(x),
$$

where $G(x)$ is the resolvent. On each link $[\alpha \beta]$, the Hamiltonian $H$ acts as the one dimensional Laplace operator $-\frac{1}{2} \Delta\left(\equiv-\frac{1}{2} \frac{\mathrm{d}^{2}}{\mathrm{~d} x_{\alpha \beta}^{2}}\right)$. Moreover, the behavior of the resolvent $G(x)$ has to be specified in the neighbourhood of all the vertices.

Consider indeed some vertex $\alpha$ and its nearest neighbours $\beta_{i}, i=1,2, \ldots, m_{\alpha}$. Suppose that the Brownian particle reaches $\alpha$. It will leave this vertex along the link $\left[\alpha \beta_{i}\right]$ with some probability $p_{\alpha \beta_{i}}$ that we may choose arbitrarily. For the sake of simplicity, we make the homogeneity assumption $p_{\alpha \beta_{i}}=1 / m_{\alpha}$. With this assumption, the resolvent $G(x)$ is shown to be continuous at all the vertices. We will denote by $G_{\alpha}$ its value on vertex $\alpha$.

If $\alpha \neq 0$, probability conservation implies

$$
\left.\sum_{i=1}^{m_{\alpha}} \frac{\mathrm{d} G}{\mathrm{~d} x_{\alpha \beta_{i}}}\right|_{x_{\alpha \beta_{i}}=0}=0 .
$$

In addition, in an infinitesimal neighbourhood of the starting vertex $O, G$ must satisfy

$$
\left(-\frac{1}{2} \Delta+\gamma+p \delta\right) G=\delta
$$

This equation is a generalization of the one dimensional case studied in Ref. [6].

Denoting by $\mu_{i}$ one of the nearest neighbours of $O$, spatial integration in this neighbourhood gives

$$
-\left.\frac{1}{2} \sum_{i=1}^{m_{0}} \frac{\mathrm{d} G}{\mathrm{~d} x_{0 \mu_{i}}}\right|_{x_{0 \mu_{i}}=0}+p G_{0}=1 .
$$

Let us now show that all the derivatives of $G$ appearing in the above equations can be expressed in terms of the $G_{\alpha}$ 's. On the link $[\alpha \beta], G\left(x_{\alpha \beta}\right)$ satisfies

$$
\left(-\frac{1}{2} \frac{\mathrm{d}^{2}}{\mathrm{~d} x_{\alpha \beta}^{2}}+\gamma\right) G\left(x_{\alpha \beta}\right)=0,
$$

whose solution is

$$
G\left(x_{\alpha \beta}\right)=G_{\alpha} \frac{\sinh \sqrt{2 \gamma}\left(l_{\alpha \beta}-x_{\alpha \beta}\right)}{\sinh \sqrt{2 \gamma} l_{\alpha \beta}}+G_{\beta} \frac{\sinh \sqrt{2 \gamma} x_{\alpha \beta}}{\sinh \sqrt{2 \gamma} l_{\alpha \beta}} .
$$

It follows that

$$
\left.\frac{\mathrm{d} G}{\mathrm{~d} x_{\alpha \beta}}\right|_{x_{\alpha \beta}=0}=-c_{\beta \alpha} G_{\alpha}+s_{\alpha \beta} G_{\beta},
$$

where

$$
\begin{aligned}
c_{\alpha \beta} & =\sqrt{2 \gamma} \operatorname{coth} \sqrt{2 \gamma} l_{\alpha \beta}=c_{\beta \alpha} \\
s_{\alpha \beta} & =\frac{\sqrt{2 \gamma}}{\sinh \sqrt{2 \gamma} l_{\alpha \beta}}=s_{\beta \alpha} .
\end{aligned}
$$


The relation (9) allows us to write Eqs. (4, 6) in a matrix form

$$
M(p) G=L,
$$

where $M(p)$ is a symmetric $(V \times V)$ matrix with elements

$$
\begin{aligned}
M_{00} & =\sum_{i=1}^{m_{0}} c_{0 \mu_{i}}+2 p \\
M_{\alpha \alpha} & =\sum_{i=1}^{m_{\alpha}} c_{\alpha \beta_{i}} \quad \text { if } \quad \alpha \neq 0 \\
M_{\lambda \mu} & =-s_{\lambda \mu} \text { if }[\lambda \mu] \quad \text { is a bond } \\
& =0 \quad \text { otherwise. }
\end{aligned}
$$

$G$ and $L$ are two $(V \times 1)$ matrices whose elements are, respectively, $G_{\alpha}$ and $L_{\alpha}=2 \delta_{\alpha 0}$. Inverting Eq. (12) we get

$$
G_{\alpha}=2\left(M(p)^{-1}\right)_{\alpha 0} .
$$

Substituting the results from Eqs. (8) and (17) in the original expression in Eq. (3) and carrying out a few elementary manipulations on determinants, we get a closed form expression for the (double) Laplace transform,

$$
\mathcal{L}=\frac{1}{\gamma} \frac{\operatorname{det} M(0)}{\operatorname{det} M(p)}
$$

To proceed further, let us denote by $\widehat{G_{\alpha}}$ the value of $G_{\alpha}$ for $p=0$. Due to the special form of the matrix $M(p)$, we may write

$$
\operatorname{det} M(p)=\left(1+p \widehat{G_{0}}\right) \operatorname{det} M(0)
$$

and recast $(18)$ in the form

$$
\int_{0}^{\infty} \mathrm{d} T e^{-p T} \int_{0}^{\infty} \mathrm{d} t e^{-\gamma t} P(T, t)=\frac{1}{\gamma} \frac{1}{1+p \widehat{G_{0}}}
$$

Since $\widehat{G_{0}}$ is independent of $p$, we may invert the Laplace transform with respect to $p$ and get the relationship

$$
\int_{0}^{\infty} \mathrm{d} t e^{-\gamma t} P(T, t)=\frac{\lambda(\gamma)}{\gamma} e^{-T \lambda(\gamma)}
$$

with

$$
\lambda(\gamma)=\frac{1}{\widehat{G}_{0}}
$$

Eq. (21) is the central result of this Letter. It provides a generalization to graphs of the Lévy formula for one dimensional diffusion processes [14]. It is worthwhile to notice that Eqs. (18,21,22) are valid for all kind of graphs irrespective of whether they are finite or not. The simplicity of this result calls for an alternative derivation based on paths integrals. The starting point is a representation of the resolvant as a two point function of a scalar field theory where the field $\phi(x)$ is a scalar field defined on the links of the graph and satisfying suitable continuity properties ( 8 ).

$$
\left\langle x\left|\frac{1}{H+\gamma}\right| 0\right\rangle=Z(p)
$$


where

$$
Z(p)=\frac{\int \mathcal{D} \phi \phi(x) \phi(0) \exp -S(\phi)}{\int \mathcal{D} \phi \exp -S(\phi)}
$$

and the action is

$$
S(\phi)=\frac{p}{2} \phi(0)^{2}+\frac{1}{2} \int \mathrm{d} x\left(\frac{1}{2}(\nabla \phi)^{2}+\gamma \phi^{2}\right)
$$

One first expands the exponential term in $p$ both in the numerator and denominator and computes all contractions using Wick's theorem. Then one can resum the series and obtain after some algebra

$$
\left\langle x\left|\frac{1}{H+\gamma}\right| 0\right\rangle=\frac{\widehat{G_{x}}}{1+p \widehat{G_{0}}} .
$$

Substituting this result in Eq. (3) and integrating over $x$, we get back the same result as in Eq. (22).

The computation of the local time distribution is therefore reduced to the calculation of the Green's function on the graph. In some cases it is quite useful to express $\lambda(\gamma)$ as a ratio of two spectral determinants of the form

$$
\lambda(\gamma)=\frac{1}{2} \frac{S_{N}(\gamma)}{S_{D}(\gamma)}
$$

where $S(\gamma)=\operatorname{det}(-\triangle+\gamma)$ is the spectral determinant of the graph. The numerator is computed with Neumann boundary conditions at all vertices and the denominator is computed with Dirichlet boundary conditions at $\mathrm{O}$ and Neumann at all other vertices. Note that such ratios of spectral determinants which arise in the context of scattering theory on graphs 8, 12, 13] have recently appeared in the mathematical litterature in relation with Dirichlet forms [11] or Sturm-Liouville problems [9].

In order to extract the behavior of the distribution $P(T, t)$ from the general formula in Eq. (21), we need to compute the function $\lambda(\gamma)$ explicitly for a given graph and then invert the Laplace transform. In general, it is not easy to invert this Laplace transform. However, in the asymptotic limit when both $t$ and $T$ large with their ratio fixed, it is possible to make progress. In this scaling limit, one expects, from generic considerations, the following scaling behavior [6. 7],

$$
P(T, t) \sim e^{-t \Phi(T / t)},
$$

where $\Phi(y)$ is a large deviation function. Substituting this expected scaling form on the left hand side of Eq. (21) and carrying out a steepest descent calculation valid for large $t$ and $T$, one obtains the following Legendre transform,

$$
\Phi(y)=\max _{\gamma}[-\gamma+y \lambda(\gamma)] .
$$

Thus the large deviation function can be computed from Eq. (29) provided one knows the function $\lambda(\gamma)$ explicitly for a given graph. Examples where one can calculate explicitly the function $\lambda(\gamma)$ are given below. Interestingly, however, some general features of the large deviation function $\Phi(y)$, such as its behaviour near the tails as well as near its minimum can be worked out for a generic graph even without the detailed knowledge of the function $\lambda(\gamma)$, thus displaying certain universal features.

First, let us consider finite graphs. One can show that $\Phi(y)$ decreases monotonically with increasing $y$ in the range $0<y<1 / l$, achieves a minimum at $y=1 / l$ and then increases monotonically for $y>1 / l$ (recall that $l$ is the total length of the graph). We consider the following three regions where the behavior of $\Phi(y)$ is quite generic. 
i) Let us first consider the limit $\gamma \rightarrow \infty$. Using Eqs. (13 16) and (19), we get

$$
\lambda(\gamma) \sim m_{0} \sqrt{\frac{\gamma}{2}}
$$

where $m_{0}$ is the coordination of the vertex $O$. Using this expression in Eq. (29) and maximizing, we get

$$
\Phi(y) \approx \frac{m_{0}^{2} y^{2}}{8} \text { when } y \rightarrow \infty
$$

The limit $y \rightarrow \infty$ means that we select infinitely long Brownian trajectories (recall that $t \rightarrow \infty$ ) for which the particle stays during almost all the time close to $O$. Since the particle does not explore the whole graph, the local time distribution will mainly depend on the characteristics of the graph in the vicinity of $O$, i.e. on the coordination $m_{0}$.

ii) By considering the first negative singularity, $\gamma_{0}$, of $\lambda(\gamma)$ in Eq. (22) we can show that, for $\gamma \approx \gamma_{0}, \lambda(\gamma) \sim a /\left(\gamma_{0}-\gamma\right)$ with $a>0$. From Eq. (29) we deduce that

$$
\Phi(y) \approx\left|\gamma_{0}\right|-2 \sqrt{a y} \quad \text { when } y \rightarrow 0^{+}
$$

where $\gamma_{0}$ is the lowest eigenvalue of the Laplacian on the graph with Dirichlet boundary conditions in $\mathrm{O}$.

iii) Finally, it turns out that $\gamma \rightarrow 0$ limit corresponds, via Eq. (29), to $y \rightarrow 1 / l$. In this limit

$$
\Phi(y) \approx \frac{1}{C}\left(y-\frac{1}{l}\right)^{2} \text { when } y \approx \frac{1}{l} .
$$

The positive constant $C$ is obtained by a small $\gamma$ expansion in Eq. (12) with the result:

$$
C=\frac{2}{l^{3}}\left|\frac{\operatorname{det} Q}{\operatorname{det} N}\right|
$$

where the matrix $N$ is obtained by removing the 1st line and 1st column in the matrix $M(0)$ and taking the limit $\gamma \rightarrow 0$. The matrix $Q$ is obtained by taking the limit $\gamma \rightarrow 0$ in the matrix $M(0)$ and replacing the 1 st line and 1st column by the elements $Q_{00}=-(1 / 3) \sum_{[\alpha \beta]} l_{\alpha \beta}^{3}$ and $Q_{0 \alpha}=Q_{\alpha 0}=\sum_{i=1}^{m_{\alpha}} l_{\alpha \beta_{i}}$ for $\alpha \neq 0$.

Note that the value $1 / l$ corresponds to the mean local time $\langle T\rangle / t$ and the constant $C$ is proportional to the variance of the local time. This result, which also follows from a central limit theorem, indicates a Gaussian distribution for the local time near its mean value, $P(T, t) \sim \exp \left[-(T-t / l)^{2} / 2 \sigma^{2}\right]$, where the variance, $\sigma^{2}=C t / 2$ for large $t$. This immediately gives a large deviation function $\Phi(y)$ as in Eq.(33). Note, however, that this central limit theorem holds only near $y \approx 1 / l$ but fails in regions away from $y=1 / l$. In particular, near the tails the distribution becomes non-Gaussian as reflected in the form of $\Phi(y)$ near $y \rightarrow 0$ and $y \rightarrow \infty$ above. For one dimensional diffusion processes, these characteristics of large deviation functions were identified not just for the local time but for other functionals of Brownian motion as well [6, 7].

As an example, consider a complete graph $K_{n}$ with $n$ vertices where there is a link of length $d$ between any pair of vertices (see fig.1). In this case, putting $p=0$ in Eq. (17), evaluating the inverse of the $M$ matrix explicitly and using this result in Eq. (22), one obtains ([8])

$$
\lambda(\gamma)=\frac{1}{2} \frac{\sqrt{2 \gamma}}{\cos (\phi)} \frac{[\cosh (d \sqrt{2 \gamma})+\cos (\phi)]}{[(\cosh (d \sqrt{2 \gamma})+\cos (\phi)-1)]} \tanh \left[\frac{d}{2} \sqrt{2 \gamma}\right]
$$




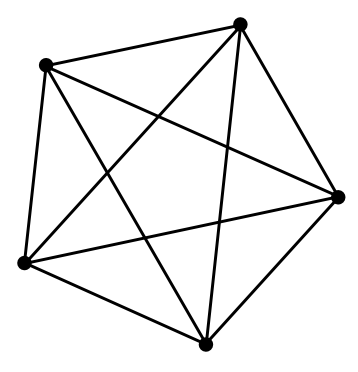

Figure 1: The complete graph $K_{5}$.

where $\cos (\phi)=\frac{1}{n-1}$. In particular, a small $\gamma$ expansion in (29) leads for the constant $C$ to the value $C=16(n+1 / n-11 / 6) /(n(n-1))^{2}$, in agreement with Eq. (34).

Another interesting example, which was recently used in the context of quantum chaos [16], 17] is the case of a star graph consisting of $n$ links of length $d$ attached to the vertex $\mathrm{O}$ (fig 2).

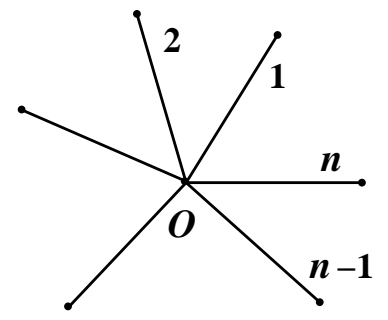

Figure 2: The hydra with $n$ legs.

In this case one obtains

$$
\lambda(\gamma)=n \sqrt{\frac{\gamma}{2}} \tanh (d \sqrt{2 \gamma})
$$

One can also compute explicitly the tails of the large deviation function and thus check that they are in agreement with the general results Eqs. (31,34). Clearly, in the latter cases ii) and iii), the whole graph is explored and the distribution depends, in a rather nontrivial way, on the details of the graph.

The previous formulae can be easily generalised to infinite graphs. The example of a star graph with infinite legs is interesting since it was already used in a probabilistic setting to derive multidimensional extensions of the arc sine law (15]). Using Eq. (36) one can derive the exact probability distribution

$$
P(T, t)=\frac{n}{\sqrt{2 \pi t}} \exp \left[-\frac{n^{2} T^{2}}{8 t}\right]
$$

The fact that the distribution is purely Gaussian depends obviously heavily on the symmetries of the graph. However the behaviour for small and large $T$ is universal. For an arbitrary graph with $n$ infinite legs attached in any vertex one can show that $\Phi(y)$ is a monotonically increasing function for $y>0$ and also that

i)

$$
\Phi(y) \approx \frac{m_{0}^{2} y^{2}}{8} \text { when } y \rightarrow \infty
$$


(same result as before and for the same reasons)

ii)

$$
\Phi(y) \approx \frac{n^{2} y^{2}}{8} \quad \text { when } \quad y \rightarrow 0
$$

(the particle tries to explore the whole graph; so, it essentially "sees" the infinite legs).

\section{References}

[1] F. Harary, Graph Theory, (Addison-Wesley, Reading, 1990).

[2] D. Sherrington and S. Kirkpatrick, Phys. Rev. Lett. 35, 1792 (1975).

[3] For a review see D. Dhar, Studying Self-Organized Criticality with Exactly Solved Models (Troisième Cycle de la Physique en Suisse Romande, Université de Lausanne, 1999), also available at cond-mat/9909009.

[4] D.M. Cvetkovic, M. Doob, and H. Sachs, Spectra of Graphs, Theory and Application (Academic, New York, 1980).

[5] J.E. Avron and L. Sadun, Ann. Phys. 206, 440 (1991).

[6] S. N. Majumdar and A. Comtet, Phys. Rev. Lett. 89, 60601 (2002).

[7] S. N. Majumdar and A.J. Bray, Phys. Rev. E 65, 051112 (2002).

[8] E. Akkermans, A. Comtet, J. Desbois, G. Montambaux and C. Texier, Annals of Physics 284, 10 (2000).

[9] A. Voros, math-phys/0201052.

[10] C. Texier and G. Montambaux, J. Phys. A Math.Gen. 3410307 (2001)

[11] C. Sabot, Ann. I.H. Poincaré PR37 275 (2001).

[12] J. Desbois, J. Phys. 33, L63 (2000).

[13] J. Desbois, Eur. Phys. J.B 15, 201 (2000).

[14] See for instance K. Itô and and H.P. McKean, Diffusion Processes and Their Sample Paths, (Springer Verlag, Berlin, Heidelberg and New York, 1974).

[15] M. Barlow, J. Pitman and M. Yor, Séminaire de Probabilités 23, Lecture Notes in Maths 1372, Springer (1989).

[16] G. Berkolaiko, E.B. Bogomolny, and J. P. Keating, J. Phys. A Math. Gen. 34335 (2001).

[17] T. Kottos and U. Smilansky, Phys. Rev. Lett. 794794 (1997). 